\title{
Ti6Al4V Thin Walls Production using Laser Directed Energy Deposition (L- DED) Process
}

\author{
Barragan, G. A., Mariani, F. E. and Coelho, R. T.

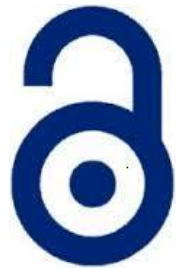 \\ Received: 26 February 2021 \\ Accepted: 29 April 2021 \\ Published: 15 July 2021 \\ Publisher: Deer Hill Publications \\ (C) 2021 The Author(s) \\ Creative Commons: CC BY 4.0
}

\begin{abstract}
One of the main applications of Directed Energy Deposition (DED) is the production of thin-wall structures, where it has significant advantages over traditional milling and machining techniques, or even welded analogues. These kinds of structures are frequently employed in aerospace components, field where titanium alloys have a primary role to play. Amongst them, the most employed is the Ti6Al4V with an alpha + beta alloy containing $6 \%$ Aluminium (Al) and $4 \%$ Vanadium $(\mathrm{V})$. It has an excellent combination of strength and toughness along with excellent corrosion resistance. For the study hereby, thin-wall structures were constructed employing a Laser Directed Energy Deposition machine (L-DED), working with powder material. Analyse identified some microstructural and mechanical characteristics, thorough metallographic study, wear test (micro-adhesive) and micro hardness test. Finding a grain refined structure with competitive mechanical properties compared to materials manufactured by traditional processes. Results positioning DED as an attractive manufacturing technology, with a huge potential to improve costs and material usage, besides almost no restriction on component shape.
\end{abstract}

Keywords: Directed Energy Deposition, Titanium, Hardness, Wear test, Mechanical properties.

\section{INTRODUCTION}

The Laser Directed energy deposition (L-DED) process allows the fabrication of components that can be used in a variety of engineering applications using a layer-by-layer strategy for constructing directly from a CAD data [1]. It employs a focused laser beam as heating source to melt in-situ delivered powder. When this occurs, a melt pool is formed on the surface of the substrate, or on a previously deposited layer. Therefore, a track is created after solidification and the process is repeated according to the planned path until completing the construction of the desired part. DED is a complicated process with many interconnected variables [2]. However, some of the advantages to justify its use are related to improved mechanical behaviour resulted in the manufactured components and its capacity to fabricate complex geometrical elements overcoming the restrictions imposed by the traditional manufacturing processes [3].

Thin wall structures comprise an important and growing proportion of engineering construction with some applications on aircraft, automobiles, turbomachinery, bridges, and machine elements where important benefits in terms of weigh reduction could be achieved [4]. When fabrication by traditional manufacturing methods shows problematical, DED has been seen a promising, advantageous and a competitive approach [5] and has being extensively explored [6-8].

The Ti6Al4 V is one of the most used alloys [9] with application at the same fields above mentioned, especially in thin-walled structures [10] due to its good mechanical properties even at high temperatures. It is also light in weight and corrosion resistant. Considering the laser additive manufacturing (AM) process the relationships between the deposition parameters, microstructure, and mechanical properties of that alloy have been studied [11] primarily with the use of unit tracks deposition onto a massive substrate [12] or the fabrication of simple geometric solid bodies. It was found improved behaviour when compared with their wrought counterparts [13] primarily due to fact that the deposited material undergoes rapid cooling enabling some grain refinements.

For these study thin wall structures were constructed employed a Laser Directed Energy (L-DED) deposition machine, working with powder material, to identify its microstructural and some mechanical behaviour, thorough

G. Barragan $1,2 \varangle$, F. E. Mariani², and R. T. Coelho ${ }^{2}$

${ }^{1}$ Grupo de Investigación en Ingeniería Aeroespacial, Universidad Pontificia Bolivariana, Circular 1 \# 70-01, Medellín, Colombia. 2LAPRAS, Sao Carlos Engineering School, University of Sao Paulo, Av. Trabalhador São Carlense 400, São Carlos, Brazil. E-mail: german.barragan@upb.edu.co 
metallographic study, wear test (micro-adhesive) and micro hardness test introducing one step further towards industrial applications.

\section{MATERIALS, METHODS AND SAMPLES ANALYSIS}

\subsection{Materials and Equipment}

For the thin wall fabrication, a plate of Ti6Al4V (substrate) and Ti6Al4V grade 5 powder supplied by GE, as the additive manufacturing feedstock were employed. The chemical composition of the powder is in accordance with ASTM F2924 - 14 [14] and is presented in Table 1. The fabrication was conducted in Magic 250 machine, this 5 axis DED system equipped with a Nd:YAC laser with a maximum nominal power of one (1) kilowatt [15].

The powder size is between $45-150 \mu \mathrm{m}$, with a flow rate of 25s measured in accordance with the ASTM B213 standard. Figure 1 (a) presents an SEM image of the powder morphology, where is possible to observe its high sphericity and low satellite formation; In Figure 1(b) a metallographic image of the powder is shown, where it is possible to observe that no internal porosities was found in the selected samples. Therefore, the powder is considered with a good quality to be used in the L-DED process.

Table 1: Chemical composition w\% - Ti6Al4V powder [16].

\begin{tabular}{cccccccc}
\hline $\mathrm{Ti}$ & $\mathrm{Al}$ & $\mathrm{V}$ & $\mathrm{Fe}$ & $\mathrm{O}$ & $\mathrm{C}$ & $\mathrm{N}$ & $\mathrm{H}$ \\
\hline $87.6-91$ & $5.5-6.75$ & $3.5-4.5$ & $\leq 0.40$ & $\leq 0.20$ & $\leq 0.08$ & $\leq 0.05$ & $\leq 0.015$ \\
\hline
\end{tabular}

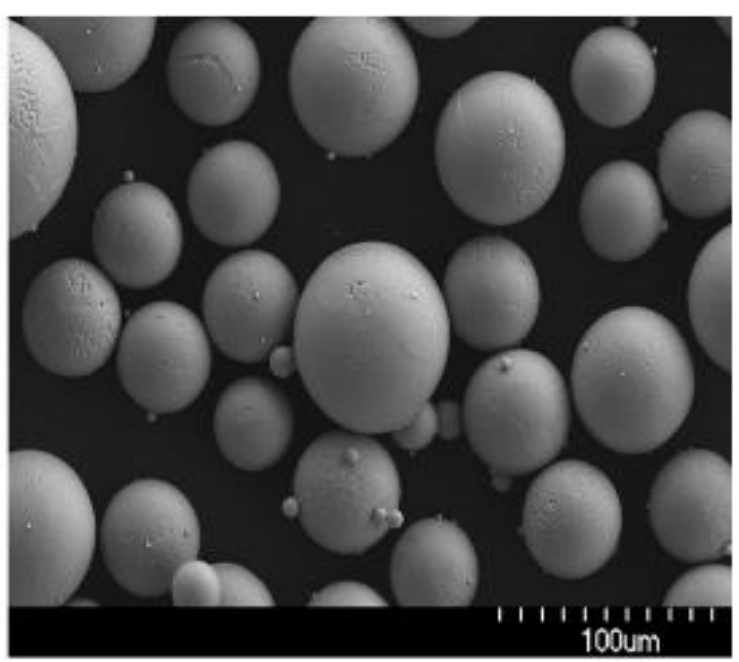

(a)

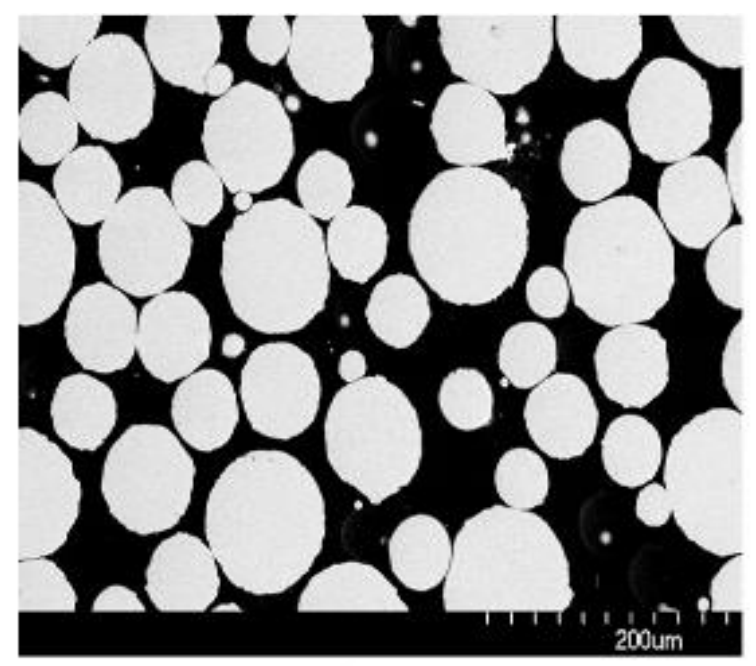

(b)

Figure 1: Ti6Al4V Powder (a) Morphology; (b) Metallographic image [16].

\subsection{Methods}

For the fabrication of the thin wall structures the process parameters listed in table 2 were employed. These parameters were selected in accordance with the machine, the material employed and previous tests to prove the consistence with an expected bead geometry. As the structure grows higher, some changes, or non-conformities, on the bead topography and geometry usually appear due to heat distribution and catchment efficiency.

After selecting the above parameters for one bead deposition, the powder flow rate for wall construction was explored. Six different thin wall structures were fabricated employing different powder flow rate as listed in Table 3. The geometry, topography and microhardness of the wall were analysed. The condition which shows the better characteristics and more homogenous shape was selected and employed for the other conducted analysis (metallographic and wear test). 
Table 2: Employed process parameters.

\begin{tabular}{lcc}
\hline & Process parameters & \\
\hline Laser Power & 300 & {$[\mathrm{~W}]$} \\
Feed Speed & 2000 & {$[\mathrm{~mm} / \mathrm{min}]$} \\
Standoff distance & 3.5 & {$[\mathrm{~mm}]$} \\
Atmosphere & Inner condition & Argon (Ar) \\
\hline
\end{tabular}

Table 3: Powder flow rates tested.

\begin{tabular}{cc}
\hline Condition & Powder flow rate $[\mathrm{g} / \mathrm{min}]$ \\
\hline a & 4,75 \\
b & 4,5 \\
c & 4,25 \\
d & 4 \\
e & 3,75 \\
f & 3,5 \\
\hline
\end{tabular}

\subsection{Sample Analysis}

Microstructures of the deposited Ti6Al4V walls were characterized using the standard metallographic procedure, which involved abrasive cutting, hand sand, polishing, and etching. Kroll's reagent $(92 \mathrm{ml}$ of distilled water, $6 \mathrm{ml}$ of $\mathrm{HNO}_{3}$, and $2 \mathrm{ml}$ of $\mathrm{HF}$ ) was used as the etching reagent. The cross-sections of the samples were analysed using a 3D laser confocal microscope LEXT 4100 by Olympus.

Vickers microhardness tests on the specimens were carried out using Model 16006300 hardness tester by Buehler, under ASTM International standard test method E384. Indentations were performed on the cross-section of the substrate and deposited walls. The tests used $3.0 \mathrm{~N}$ load and a dwell time of $15 \mathrm{~s}$ to obtain the microhardness profiles.

Micro-adhesive wear tests were performed deposited walls of Ti6Al4V. The tested surfaces were ground, and polishing. The "calotest" test was then performed using a quenched and tempered AISI 52100 steel ball, with 60 HRC hardness and diameter of $25.4 \mathrm{~mm}$, driven by the driving shaft, sliding against the sample, with $1.7 \mathrm{~N}$ load. The sphere rotation speed was $275 \mathrm{rpm}$. The test times were 5, 10, 15, 20, 30 and $40 \mathrm{~min}$ for each condition studied, resulting in sliding distances of 110,220,330,440,600 and $880 \mathrm{~m}$, respectively. The volume of material removed (V) was calculated according to Equation 1:

$$
V=\pi d 4 / 64 R ; \text { for } d<<R
$$

where $d$ represents the diameter of worn caps, measured by optical microscope and $\mathrm{R}$ represents the radius of the sphere [17].

The wear tests were carried out at $29^{\circ} \mathrm{C}$ and a relative humidity of $49 \%$, under dry sliding friction (in air and without lubrication), to prevent any interfacial element from causing influences on the effect of microstructural features.

\section{RESULTS AND DISCUSSION}

Figure 2 shows different walls fabricated, where it is possible to see some topography defects on many of them caused for incorrect selections of the powder flow rate.

Figure 3 shows the Vickers microhardness profiles obtained for the deposited walls. These tests were conducted for all the specimens to identify the influence of powder flow rate on the wall shape. For all walls, it appears that hardness remains constant from the top until the interface with the substrate. Values were all close to $400 \mathrm{HV}_{3.0}$, except condition (a), Table 3, which went slightly higher, close to $450 \mathrm{HV}_{3.0}$. At the interface with substrate, at the dilution region and heat affected zone (HAZ), the hardness declines abruptly, remaining in the substrate with values close to 330 HV3.0. The average hardness values of the layers were similar, regardless of the deposition parameters. 

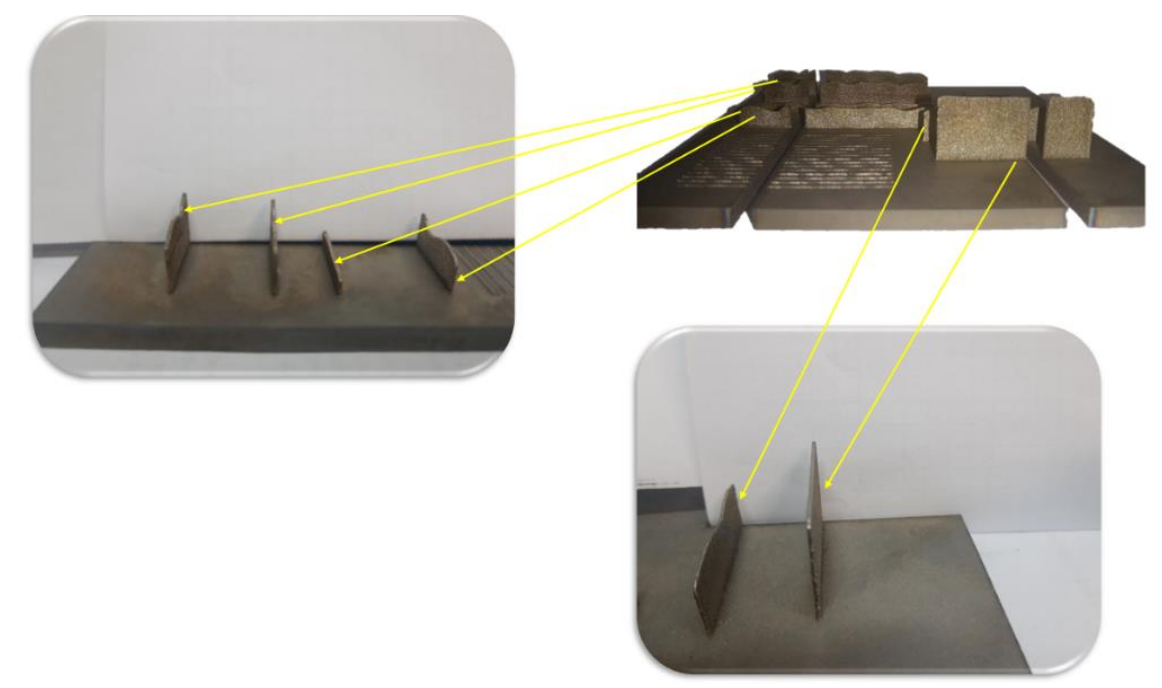

Figure 2: Fabricated walls

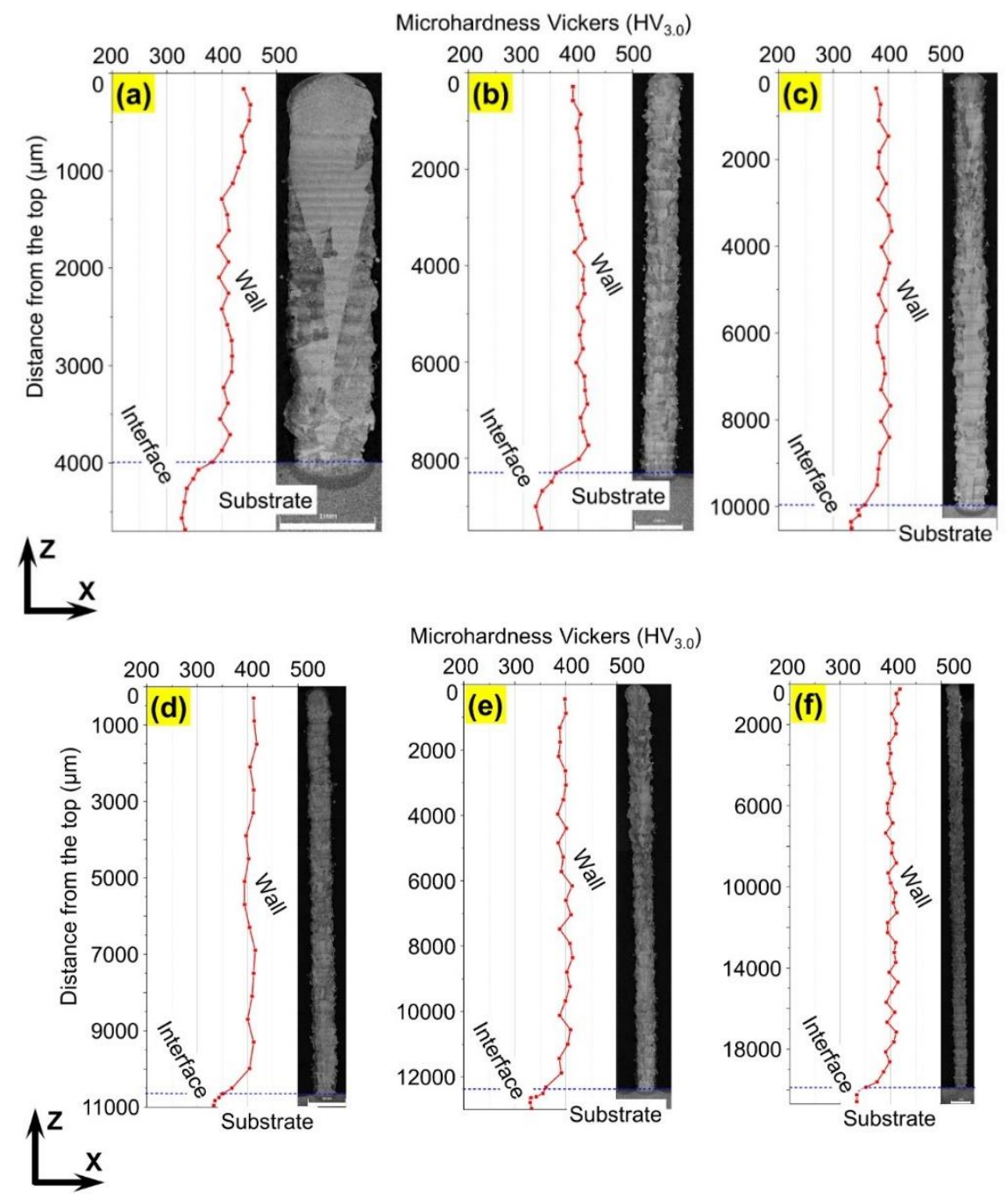

Figure 3: Vickers microhardness profiles. Conditions according to Table 3. Etching: Kroll's Reagent. 
Figure 4 shows the image of the wall (f) in Table 3, which presents the best resulted shape and was selected to conduct the metallographic and wear test. Figure 5 shows the optical micrograph of the Ti6Al4V substrate, with an average hardness of $330 \pm 2$ HV3.0. It can be noticed the presence of hexagonal close packed $\alpha$-phase and bodycentred cubic $\beta$-phase structures [18,19]. Figure 6 shows the optical micrograph of the longitudinal section of the selected wall (f).

The deposition pattern is verified layer by layer, indicated by the deposition trajectory (blue arrows). The distance between boundaries of layers is $200 \mu \mathrm{m}$, consistent with the parameters used for deposition. Microstructure of columnar dendrites was observed, which grew epitaxially from the substrate (direction Z), with growth direction opposite to the direction of heat flow. During DED deposition, the substrate and/or the deposited layer act as heat sinks, which allows the growth of the grains to be against the direction of the heat flow [20]. Figure 7 shows the micro-adhesive wear graphs obtained for the Ti6Al4V substrate of the selected wall (f).

Compared to the substrate, the wear resistance of the deposited selected wall, condition (f) Table 3, initially presented a better performance. During the tests there was a convergence behaviour, and, at the end, the performances were very close. The wall showed wear resistance $1.1 \times$ higher when compared to the substrate.

The optical micrographs of the worn caps produced by the micro-adhesive wear test are shown in Figure 8 . The analysed caps refer to the longest sliding distance (test time $40 \mathrm{~min}-880 \mathrm{~m}$ sliding distance).

For the substrate and wall, the wear surface indicated two active wear mechanisms. The first type of abrasion is two bodies (indicated by red arrows), with the presence of directional scratches in the direction of sliding of the sphere. This wear mechanism occurred through the particles of the material itself that detach and adhere to the sphere [21]. The second mechanism observed is the adhesive (indicated by yellow arrows), represented by the transferred metal films. The same characteristics were observed throughout other similar tests performed in different places of the same pieces.

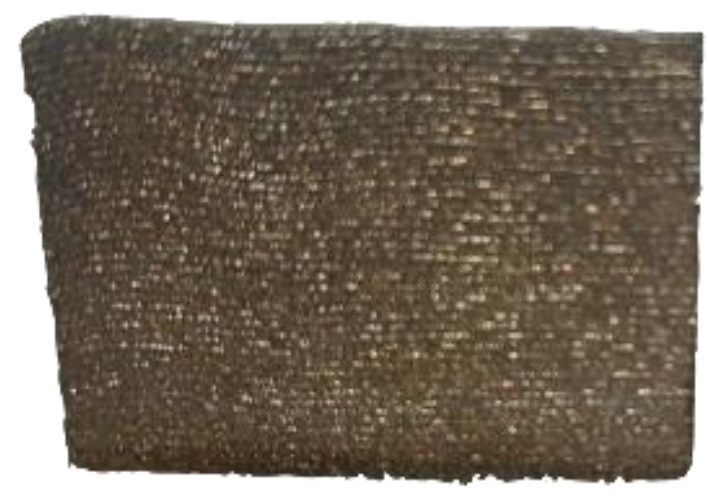

Figure 4: Selected wall (f) from Table 3

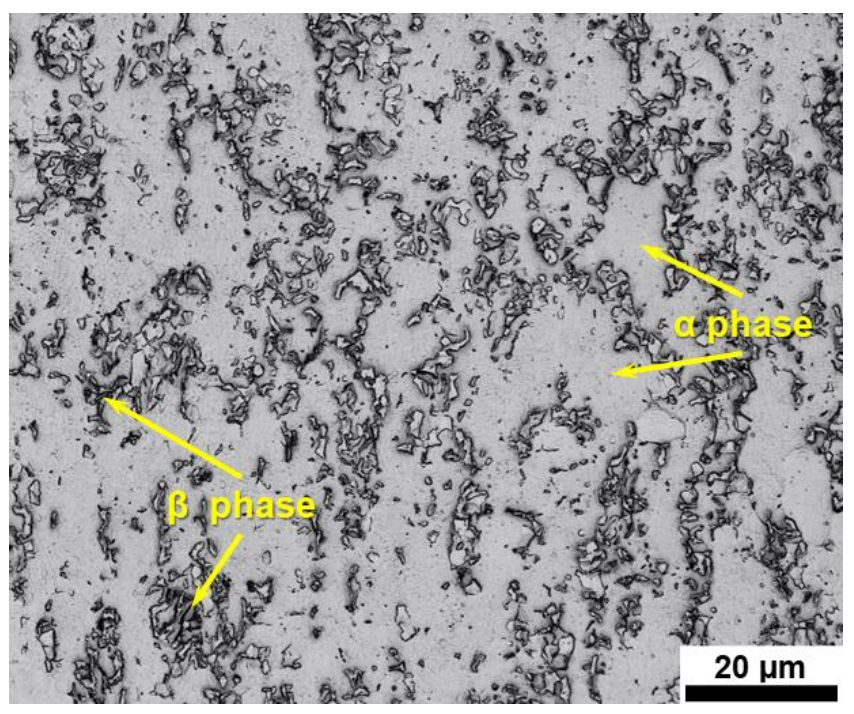

Figure 5: Optical micrograph of the as-built Ti6Al4V alloy. $\alpha$ and $\beta$ phases highlighted with yellow lines. Etching: Kroll's Reagent. 


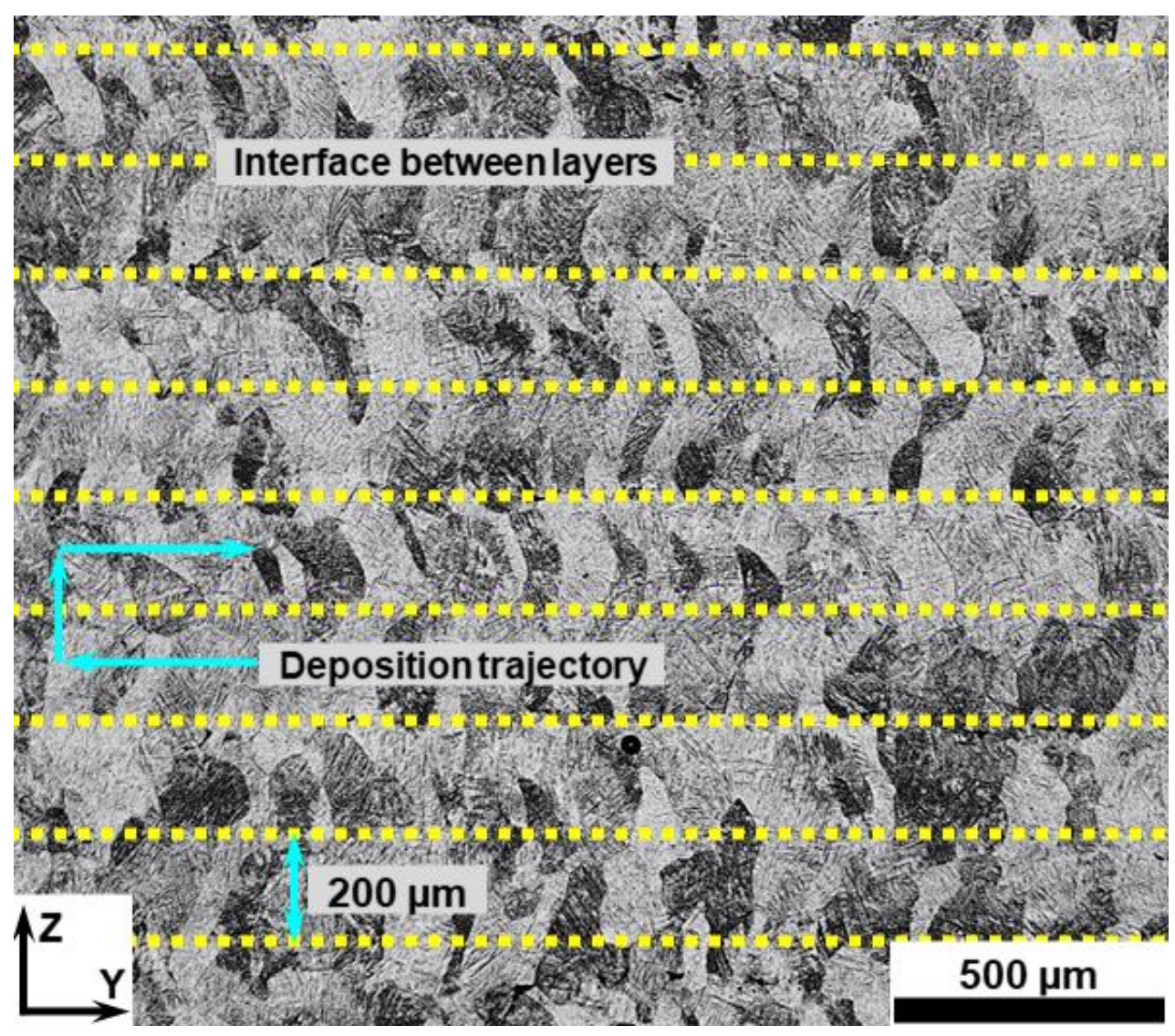

Figure 6: Longitudinal section of the WALL (f) Etching: Kroll's Reagent.

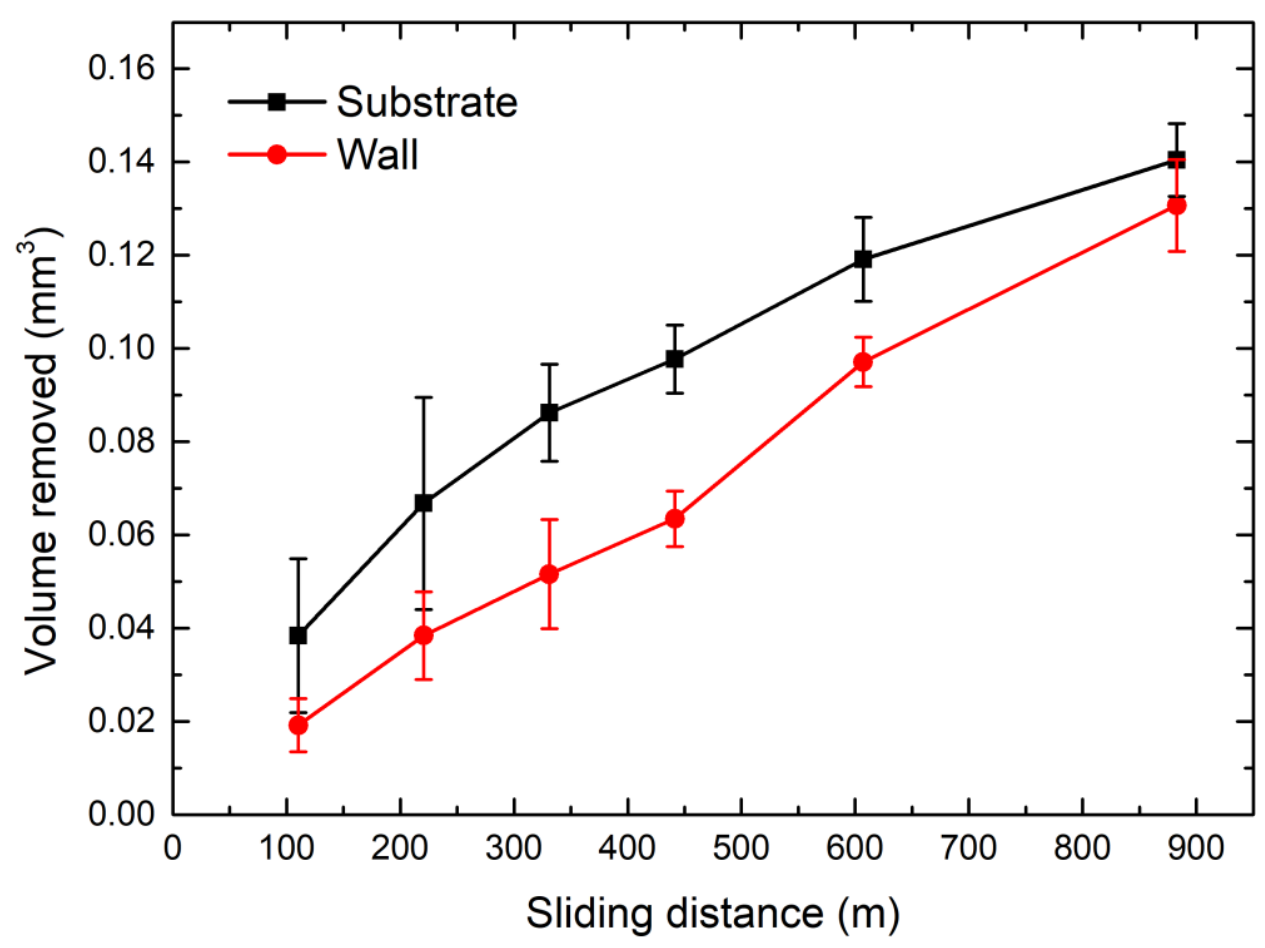

Figure 7: Micro-adhesive wear graphics obtained for the substrate wall (f). 


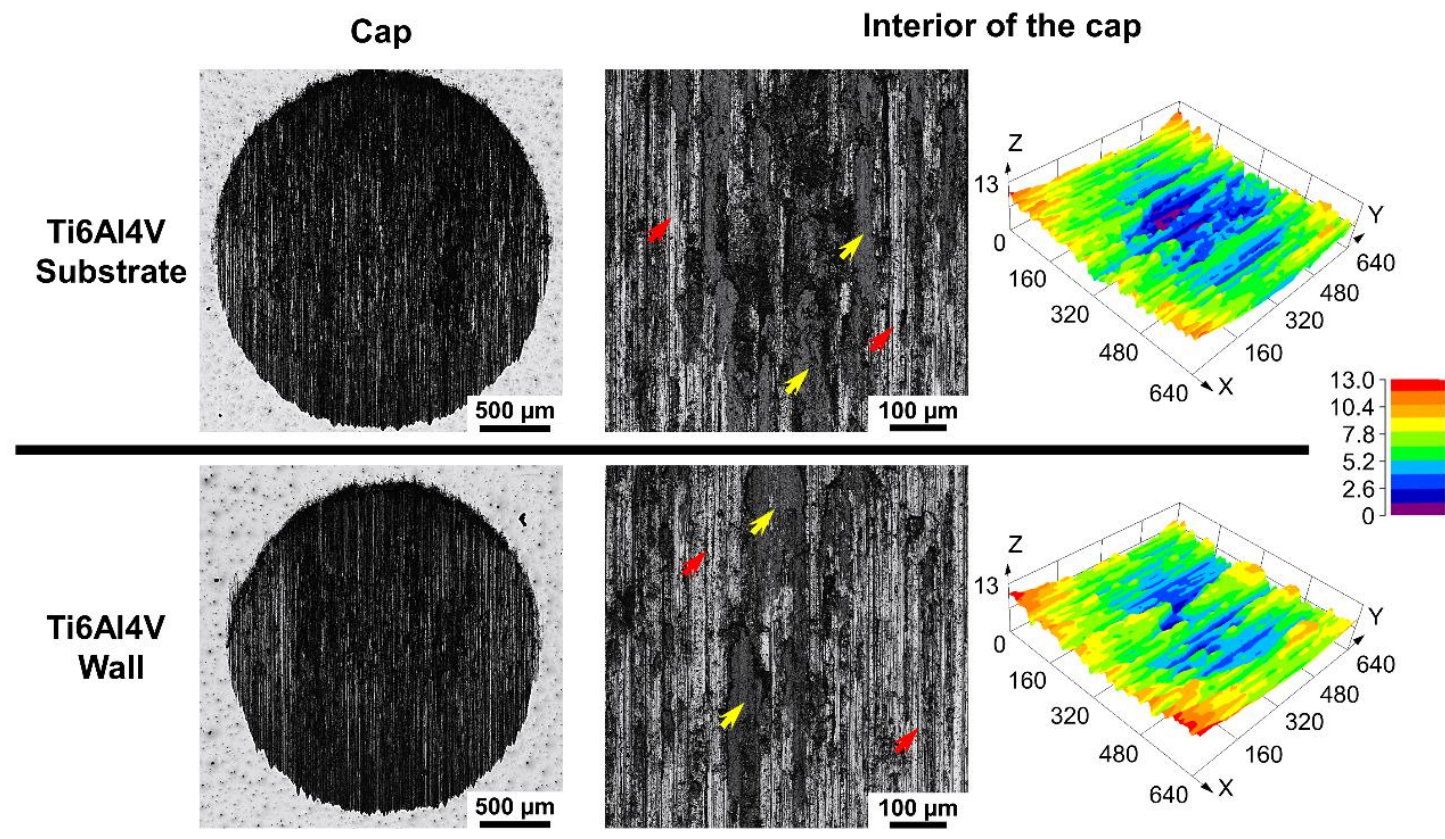

Figure 8: Optical micrograph of the wear caps and interior of the wear caps from the substrate and WALL (f). Travelled distance: $880 \mathrm{~m} \mathrm{(40} \mathrm{min).} \mathrm{The} \mathrm{yellow} \mathrm{and} \mathrm{red} \mathrm{arrows} \mathrm{indicate} \mathrm{the} \mathrm{mechanisms} \mathrm{of} \mathrm{adhesive} \mathrm{and} \mathrm{abrasive}$ wear on two bodies, respectively.

\section{CONCLUSIONS}

Investigation on the characteristics of Ti6Al4V thin-walled structures with respect to dimensional characteristics, hardness and wear behaviour has been carried out, and based upon the findings from the experimental work the following conclusions can be drawn:

1. The use of the DED process for manufacturing thin walls with Ti6Al4V showed to be feasibly and presented good results, in terms of shape and geometry of the specimens.

2. Due to the nature of the process that involves rapid cooling grain refinement appeared on the deposited walls.

3. For all walls, the hardness remains constant from the top to the interface, with values close to $400 \mathrm{HV3.0.}$

4. Harness values shown an important improvement over wrought material, used as substrate.

5. The wear resistance of the best deposited wall, shown a better performance, during the wear tests with values around $10 \%$ higher.

\section{ACKNOWLEDGEMENT}

The authors would like to appreciate the financial support of the São Paulo Research Foundation - (FAPESP) - grants 2016/11309-0; 2019/26362-2 and Coordenação de Aperfeiçoamento de Pessoal de Nível Superior - Brazil (CAPES) Finance Code 001.

\section{REFERENCES}

1. Ghosal, P., Majumber, M. C., \& Chattopadhyay, A. (2018). Study on direct laser metal deposition. Materials Today: Proceedings, 5(5), 12509-12518. doi: 10.1016/j.matpr.2018.02.232

2. Mahamood, R. (2018). Laser Metal Deposition of Metals and Alloys. Springer. doi:10.1007/978-3-319-6498565

3. Dass, A., \& Moridi, A. (2019). State of the Art in Directed Energy Deposition: From Additive Manufacturing to Materials Design. Coatings, 9(7), 418. doi: 10.3390/coatings9070418

4. Wang, F., Cheng, X., Zheng, G. M., Yang, X. H., Guo, Q. J., \& Sun, Q. L. (2019). Study of micromilling parameters and processes for thin wall fabrications. Precision Engineering, 56, 246-254. doi: 10.1016/j.precisioneng.2018.12.005

5. Jinoop, A. N., Paul, C. P., Nayak, S. K., Kumar, J. G., \& Bindra, K. S. (2021). Effect of laser energy per unit powder feed on Hastelloy-X walls built by laser directed energy deposition based additive manufacturing. Optics \& Laser Technology, 138, 106845. doi: https://doi.org/10.1016/j.optlastec.2020.106845 
6. Wu, Y., \& Yang, L. (2021). Elastic and failure characteristics of additive manufactured thin wall lattice structures with defects. Thin-Walled Structures, 161, 107493. doi: 10.1016/j.tws.2021.107493

7. Jinoop, A. N., Paul, C. P., Mishra, S. K., \& Bindra, K. S. (2019). Laser Additive Manufacturing using directed energy deposition of Inconel-718 wall structures with tailored characteristics. Vacuum, 270-278. doi: 10.1016/j.vacuum.2019.05.027

8. Demier, A. G. (2018). Micro laser metal wire deposition for additive manufacturing of thin-walled structures. Optics and Lasers in Engineering, 100, 9-17. doi: 10.1016/j.optlaseng.2017.07.003

9. Banerjee, D., \& Williams, J. C. (2013). Perspectives on Titanium Science and Technology. Acta Materialia, 61(3), 844-879. doi: 10.1016/j.actamat.2012.10.043

10. Kranz, J., \& Herzog, D. (2015). Design guidelines for laser additive manufacturing of lightweight structures in TiAl6V4. Journal of Laser Applications, 27, S14001. doi: 10.2351/1.4885235

11. Du, F., Zhu, J., Ding, X., Zhang, Q., Ma, H., Yang, J., . . Fan, S. (2019). Dimensional characteristics of Ti-6Al$4 \mathrm{~V}$ thin-walled parts prepared by wire-based multi-laser additive manufacturing in vacuum. Rapid Prototyping Journal. doi: 10.1108/RPJ-08-2018-0207

12. Niz'ev, V. G., Khomenko, M. D., \& Mirzade, F. K. (2018). Process planning and optimisation of laser cladding considering hydrodynamics and heat dissipation geometry of parts. Quantum Electronics, 48(8), 743-748. doi:10.1070/qel16708

13. Ren, Y. M., Lin, X., Yang, H. O., Tan, H., Chen, J., Jian, Z. Y., . . Huang, W. D. (2021). Microstructural features of Ti-6Al-4V manufactured via high power laser directed energy deposition under low-cycle fatigue. Journal of Materials Science \& Technology, 83, 18-33. doi: 10.1016/j.jmst.2020.12.026

14. ASTM International. (2014). Standard Specification for Additive Manufacturing Titanium-6 Aluminum-4 Vanadium with Powder Bed Fusion. West Conshohocken, PA. doi:10.1520/F2924-14

15. AddUP. (2021, 1 15). BeAM Machines. Retrieved 12 20, 2020, from https://www.beammachines.com/products/modulo250

16. General Electric. (2021, 2 25). Retrieved from AP\&C a GE Additive company: https://www.advancedpowders.com/

17. Alfaia, M. A., Oliveira, R., Lima, T. S., Mariani, F. E., Casteletti, L. C., Cheung, N., \& Garcia, A. (2020). Effects of cooling rate and microstructure scale on wear resistance of unidirectionally solidified $\mathrm{Al}-3.2 \mathrm{wt} . \% \mathrm{Bi}-(1 ; 3)$ wt.\%Pb alloys. Materials Today Communications, 25, 101659. doi: 10.1016/j.mtcomm.2020.101659

18. Patel, P., Jamnapara, N. I., Zala, A., \& Kahar, S. D. (2020). Investigation of hot-dip aluminized Ti6Al4V alloy processed by different thermal treatments in an oxidizing atmosphere. Surface and Coatings Technology, 385, 125323. doi: 10.1016/j.surfcoat.2019.125323

19. Arrazola, P. J., Garay, A., Iriarte, L. M., Armendia, M., Mayra, S., \& Le Maitre, F. (2009). Machinability of titanium alloys (Ti6Al4V and Ti555.3). Journal of Materials Processing Technology, 209(5), 2223-2230. doi: 10.1016/j.jmatprotec.2008.06.020

20. Dinda, G. P., Dasgupta, A. K., \& Mazumder, J. (2009). Laser aided direct metal deposition of Inconel 625 superalloy: Microstructural evolution and thermal stability. Materials Science and Engineering: A, 509(1-2), 98104. doi: 10.1016/j.msea.2009.01.009

21. Budinski, K. G. (2013). Friction, Wear, and Erosion Atlas. Boca Raton: CRC Press. doi: 10.1201/b15984 